\title{
El Aprendizaje Orientado a Proyectos a través de la Elaboración de una Revista Profesional: Aplicación a la Asignatura de "Auditoría de Cuentas"
}

\author{
Javier Montoya-del-Corte ${ }^{a}$, Estefanía Palazuelos-Cobo ${ }^{b}$, Ana Fernández-Laviada ${ }^{c}$ y \\ Paula San-Martín-Espinad \\ aUniversidad de Cantabria, javier.montoya@unican.es; ${ }^{b}$ Universidad de Cantabria, \\ estefania.palazuelos@unican.es; ${ }^{c}$ Universidad de Cantabria, ana.fernandez@unican.es; ${ }^{d}$ Universidad \\ de Cantabria, paula.sanmartin@unican.es
}

\begin{abstract}
In this paper the application of Project-Oriented Learning (POL) on the subject of "Auditing" of $4^{\text {th }}$ year of the Degree in Business Administration is addressed. The project attended by the 147 participating students consisted in the development and public exposure of a professional spreading journal about the subject under study. The obtained results are based on the analysis of the learning evidences gathered, the assessment tools used, the responses to a circularized questionnaire to participants at the end of the project and the ideas derived from a subsequent discussion group composed of students and professors. Among the main attained conclusions, the suitability of POL is noteworthy, on the terms described in this paper, for students to strengthen certain relevant professional skills in the studied discipline, to encourage their attitude to innovation and creativity, to give value to the effort made and to know how to deal with the completion of various and long-haul tasks with support in collaborative work.
\end{abstract}

Keywords: Project-Oriented Learning, Professional competences, Professional skills, Creativity, Assessment rubrics, Peer assessment. 
El Aprendizaje Orientado a Proyectos a través de la Elaboración de una Revista Profesional: Aplicación a la Asignatura de "Auditoría de Cuentas"

\begin{abstract}
Resumen
En este trabajo se aborda la aplicación del Aprendizaje Orientado a Proyectos (AOP) en la asignatura de "Auditoría de cuentas" de $4^{\circ}$ de Grado en Administración y Dirección de Empresas. El proyecto desarrollado por los 147 estudiantes participantes consistió en la elaboración y exposición pública de una revista de divulgación profesional sobre la materia objeto de estudio. Los resultados obtenidos están basados en el análisis de las evidencias de aprendizaje recabadas, los instrumentos de evaluación utilizados, las respuestas a un cuestionario circularizado a los participantes al finalizar el proyecto y las ideas derivadas de un subsiguiente grupo de discusión integrado por estudiantes y profesores. Entre las principales conclusiones alcanzadas cabe destacar la idoneidad del AOP, en los términos descritos en este trabajo, para que los estudiantes desarrollen competencias profesionales relevantes en la disciplina estudiada, fomenten su capacidad de innovación y creatividad, pongan en valor el esfuerzo realizado y sepan enfrentarse a la realización de tareas diversas y de largo recorrido con apoyo del trabajo colaborativo.
\end{abstract}

Palabras clave: Aprendizaje Orientado a Proyectos, Competencias profesionales, Habilidades profesionales, Creatividad, Rúbricas de evaluación, Coevaluación.

\title{
Introducción
}

Desde el inicio del proceso de implantación del Espacio Europeo de Educación Superior (EEES), los miembros del Grupo Docente de Contabilidad de la Universidad de Cantabria venimos formándonos continuadamente en distintos métodos de enseñanza y técnicas didácticas pertinentes para el adecuado desarrollo de competencias profesionales en nuestros estudiantes de Grado y Posgrado. Una de las innovaciones educativas que más esfuerzo nos conlleva cada año, pero a la vez que mayor impacto creemos que genera entre los estudiantes, está basada en el Aprendizaje Orientado a Proyectos (AOP). El aprendizaje experiencial y reflexivo que aporta a los estudiantes el desarrollo del conjunto de actividades que a continuación se explican, supone un importante paso al frente en el proceso de adaptación de la enseñanza de la Contabilidad, en general, y la Auditoría de cuentas, en particular, al citado EEES y a las necesidades del entorno y del mundo de los negocios en la actualidad.

Dentro de las disciplinas aludidas, son un importante referente las International Education Standards (IES), emitidas por el International Accounting Education Standards Board 
(IAESB) de la International Federation of Accountants (IFAC). Este marco normativo define competencia profesional como "la capacidad de una persona para ejecutar un trabajo cumpliendo con un determinado estándar en entornos laborales reales". Asimismo, define capacidad como "el conjunto formado por los conocimientos, habilidades, y valores, ética y actitudes profesionales requeridos para demostrar competencia” (IFAC, 2014, pp. 20-21). De estas dos definiciones se desprende que, para ser competentes, los profesionales deben estar capacitados desde tres vertientes diferenciadas: cognitiva, procedimental y actitudinal. Centrando la atención en el campo específico de la Auditoría de cuentas, es preciso acudir a la "IES 8: Competence Requirements for Audit Professionals". Esta norma establece el conjunto de conocimientos (párrafos 32 a 41), habilidades (párrafos 42 a 44) y valores, ética y actitudes (párrafos 45 a 52) requeridos para ser competente en el ejercicio de esta actividad profesional. Algunos de estos elementos están presentes en la literatura previa que estudia la formación en competencias de los futuros profesionales de la Auditoría de cuentas.

En los últimos años se ha incrementado notablemente el número de investigaciones dirigidas a profundizar en el estudio de las competencias profesionales requeridas en Contabilidad y Auditoría de cuentas. Existe evidencia empírica que sustenta que los empleadores que operan en el mercado laboral consideran que los titulados universitarios no están suficientemente preparados en algunas de las competencias profesionales requeridas (Kavanagh y Drennan, 2008; Frecka y Reckers, 2010; Hancock et al., 2010; Daff et al., 2012; Pan y Perera, 2012; Tempone et al.; 2012; Lin et al., 2013; Senik et al., 2013). Para corregir esta brecha formativa, algunas de las estrategias sugeridas en la literatura incluyen la adquisición de experiencia profesional práctica durante el Grado (Paisey y Paisey, 2010) y la obtención y desarrollo de competencias genéricas y transversales para la mejora de la empleabilidad (Stoner y Milner, 2010). Ahora bien, la propuesta de actuación más extendida es la que apoya un papel más activo por parte de los profesores universitarios por medio de intervenciones educativas innovadoras (véase Apostolou et al., 2010 y 2013).

\section{Objetivos}

El objetivo general de este trabajo es evaluar la idoneidad de la aplicación del Aprendizaje Orientado a Proyectos (AOP) como metodología activa en las enseñanza de Grado bajo las premisas del Espacio Europeo de Educación Superior (EEES). Como objetivos específicos se plantean los siguientes:

$1^{\circ}$ ) Determinar si el AOP favorece el desarrollo de competencias profesionales relevantes y, en tal caso, qué habilidades o destrezas permite fortalecer en mayor medida.

$2^{\circ}$ ) Averiguar si la concesión de cierto grado de libertad a los estudiantes en el desarrollo del proyecto repercute positivamente en su capacidad de innovación y creatividad.

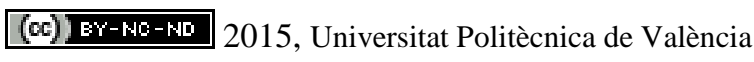

Congreso In-Red (2015) 
El Aprendizaje Orientado a Proyectos a través de la Elaboración de una Revista Profesional: Aplicación a la Asignatura de "Auditoría de Cuentas"

$3^{\circ}$ ) Examinar si la utilización de rúbricas de evaluación y coevaluación contribuye a que los estudiantes pongan en valor el esfuerzo realizado.

$4^{\circ}$ ) Indagar qué aspectos novedosos de esta aplicación concreta del AOP son valorados positiva y negativamente por los estudiantes.

\section{Desarrollo de la innovación}

\subsection{Asignatura y participantes}

La experiencia educativa que a continuación se explica se lleva a cabo dentro de la asignatura “Auditoría de cuentas”, impartida durante el primer cuatrimestre de $4^{\circ}$ del Grado en Administración y Dirección de Empresas (GADE) de la Universidad de Cantabria. Esta asignatura es de carácter obligatorio y tiene asignados 6 créditos ECTS. En el presente curso académico 2014-15 tiene matriculados 194 estudiantes repartidos en cuatro grupos, dos de mañana (de 9 a 11 y de 11 a 13 horas) y dos de tarde (de 15 a 17 y de 17 a 19 horas). De ellos, participan en todas las actividades propuestas dentro de este proyecto un total de 147 estudiantes (75,8\%), que son los que se toman como referencia para el posterior análisis de resultados. En la Tabla 1 se presenta el perfil de los estudiantes participantes.

Tabla 1. Perfil de los estudiantes participantes en la experiencia educativa

\begin{tabular}{|c|c|}
\hline Atributo & Reparto \\
\hline Sexo & $\begin{array}{l}\text { Mujeres: } 54,4 \% \\
\text { Hombres: } 45,6 \%\end{array}$ \\
\hline Edad & $\begin{array}{l}\text { Mínima: } 21 \text { años } \\
\text { Máxima: } 33 \text { años } \\
\text { Media: } 23 \text { años }\end{array}$ \\
\hline Años de estudio en la universidad & $\begin{array}{l}\text { Mínimo: } 4 \text { años } \\
\text { Máximo: } 8 \text { años } \\
\text { Media: } 5 \text { años }\end{array}$ \\
\hline $\begin{array}{l}\text { Número de asignaturas pendientes } \\
\text { para finalizar el Grado }\end{array}$ & $\begin{array}{l}\text { Mínimo: } 1 \\
\text { Máximo: } 15 \\
\text { Media: } 9\end{array}$ \\
\hline Nota media del expediente & $\begin{array}{l}\text { Aprobado: } 68,5 \% \\
\text { Notable: } 31,5 \%\end{array}$ \\
\hline
\end{tabular}

Fuente. Elaboración propia. 


\subsection{Proyecto a realizar}

Para lograr el objetivo de contribuir al desarrollo de competencias profesionales relevantes en Auditoría de cuentas, se recurre al AOP. Este método de enseñanza promueve el trabajo autónomo de los estudiantes a través de la planificación, diseño y ejecución de una serie de tareas y actividades, con la puesta en práctica de los aprendizajes adquiridos y el uso efectivo de recursos diversos. Además, entran en juego otros métodos de enseñanza secundarios, fundamentalmente el aprendizaje cooperativo, el aprendizaje basado en la investigación y el estudio de casos.

El proyecto a realizar por los estudiantes consiste en la elaboración de una revista de divulgación profesional sobre Auditoría de cuentas, que se desarrolla en un período aproximado de cuatro meses durante la impartición de la asignatura. La revista se elabora en equipos formados al inicio del cuatrimestre por los propios estudiantes. En total se establecen 35 equipos integrados por 3-5 personas.

La revista debe tener tres contenidos determinados de antemano por los profesores:

1. Una entrevista personal con un profesional de la auditoría de cuentas.

2. Un artículo sobre la evolución de la situación de la Auditoría de cuentas en España en los últimos cinco años.

3. Un artículo sobre los últimos escándalos financieros ocurridos en España en los que se hayan visto involucrados auditores de cuentas.

Además, debe complementarse con todo aquello que los estudiantes consideren oportuno introducir libremente y que sea acorde con los contenidos de la materia, como pueden ser: entrevistas a otro tipo de profesionales, artículos sobre otros temas de actualidad, noticias de actualidad comentadas, ofertas de empleo, becas de prácticas, cursos formativos, pasatiempos y publicidad.

\subsection{Evaluación del proyecto}

Durante el cuatrimestre se dedican un total de 6 horas lectivas en clase a seminarios o talleres relacionados con el desarrollo de este proyecto. Asimismo, se dispone de 4 horas de tutorías semanales. El objetivo es dedicar un tiempo de atención y seguimiento personalizado a cada equipo, que sirva para aclarar dudas y construir aprendizaje mediante la interacción entre estudiantes y equipo docente de la asignatura, de tal forma que estos asumen el rol de instructor, orientador, facilitador y motivador. 
El peso del proyecto en la calificación final de la asignatura es de un $40 \%$, repartido de la siguiente manera: 30\% los contenidos de la revista y 10\% la exposición de la misma. El otro $60 \%$ se evalúa de forma continua a través de tres exámenes parciales de teoría y práctica.

Con relación a la evaluación del proyecto, en la que participa todo el equipo docente de la asignatura, cabe destacar tres aspectos fundamentales:

1. Se califican a través de rúbricas tanto los contenidos incluidos en la revista como la exposición pública de la misma.

2. La evaluación de los contenidos se realiza en dos momentos. Una primera, antes de la exposición de la revista, que se comenta con los propios estudiantes. Y una segunda, a los 15 días de haber expuesto la revista, una vez atendidos los comentarios y observaciones incorporadas en las rúbricas.

3. En la evaluación también participan los propios estudiantes, por medio de la coevaluación a cada uno de los integrantes de su equipo.

Los contenidos de la revista se valoran sobre 10 puntos:

- Entrevista al auditor de cuentas: 2,5 puntos.

- Artículo sobre la situación de la Auditoría de cuentas: 2,5 puntos.

- Artículo sobre escándalos financieros: 2 puntos.

- Contenido libre: 2 puntos.

- Diseño y formato de la revista: 1 punto.

La exposición de la revista también se valora sobre 10 puntos:

- Creatividad: 5 puntos.

- Confianza: 3 puntos.

- Participación: 1 punto.

- $\quad$ Ajuste al tiempo disponible: 1 punto.

En las Figuras 1 y 2 se muestran, respectivamente, las portadas de algunas revistas elaboradas por los estudiantes e imágenes de algunas exposiciones realizadas. 
Javier Montoya-del-Corte, Estefanía Palazuelos-Cobo, Ana Fernández-Laviada y Paula SanMartín-Espina

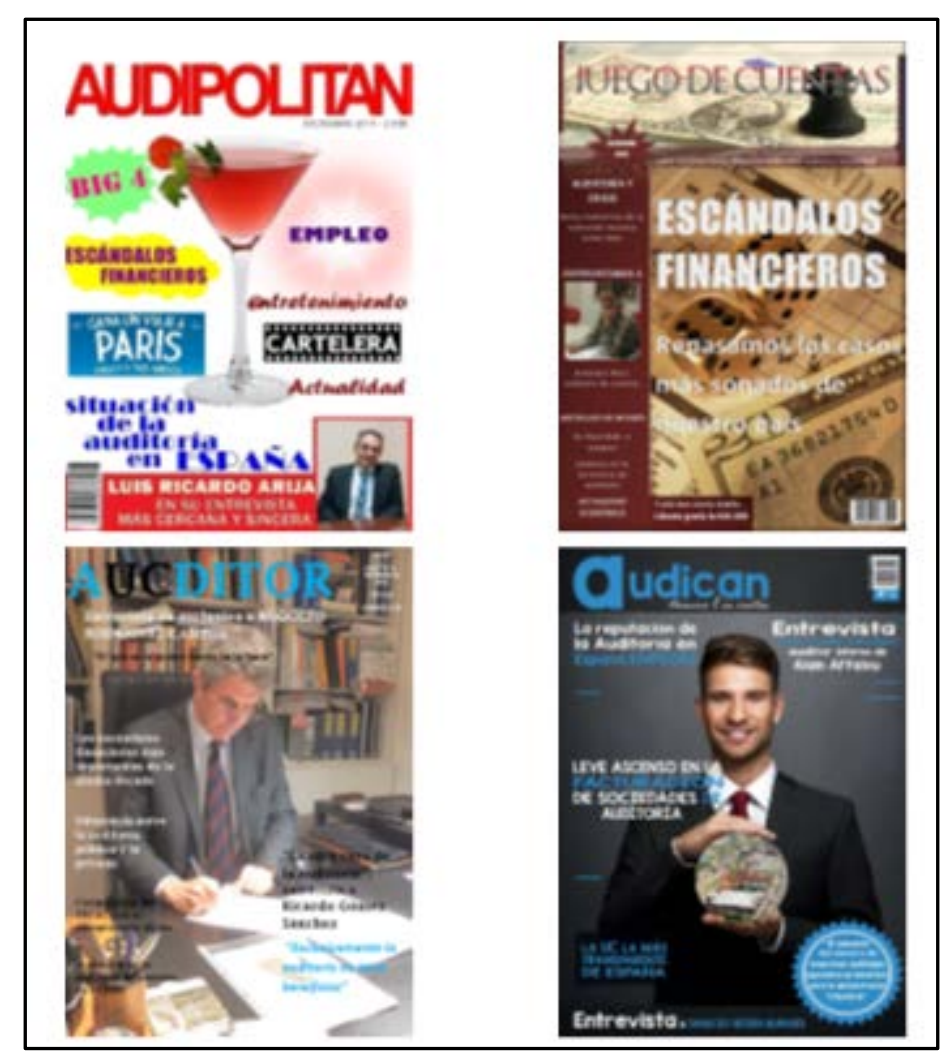

Fig. 1 Ejemplos de revistas elaboradas por los estudiantes

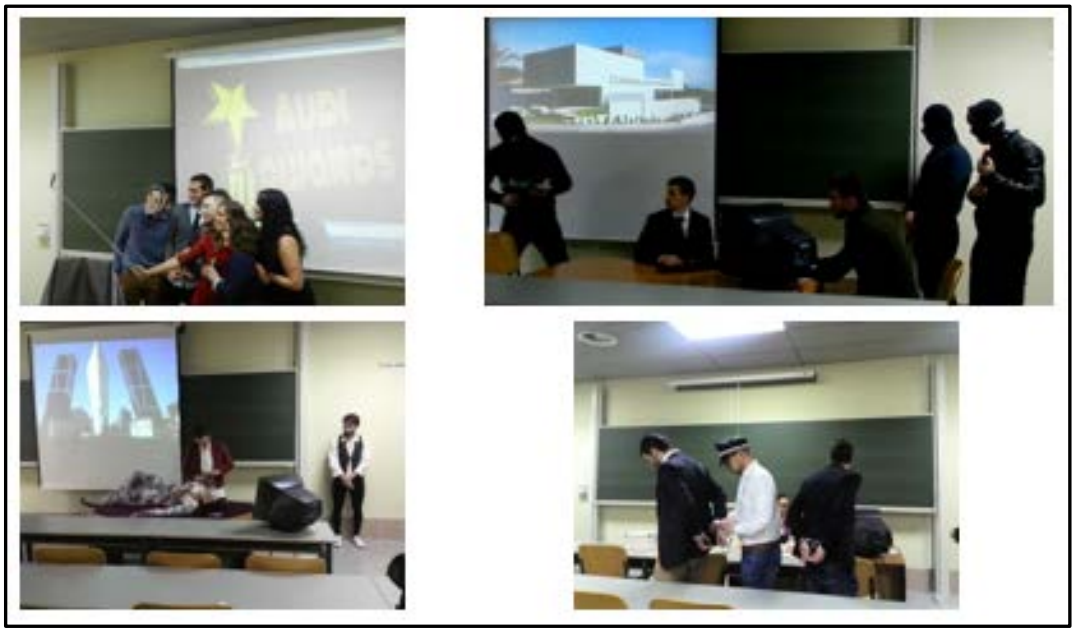

Fig. 2 Ejemplos de exposiciones realizadas por los estudiantes

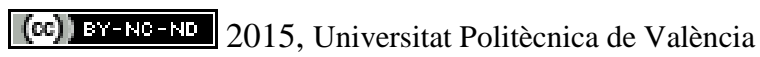

Congreso In-Red (2015) 
El Aprendizaje Orientado a Proyectos a través de la Elaboración de una Revista Profesional: Aplicación a la Asignatura de "Auditoría de Cuentas"

Además, en los siguientes enlaces pueden visualizarse las grabaciones de las exposiciones de tres de los equipos, con las que principalmente se persigue promover la creatividad de los estudiantes:

- Exposición a través de una “Misa”: http://youtu.be/RPHQOgN2lyc

- Exposición a través de un "Debate televisivo": http://youtu.be/X6wSeW-R7B8

- Exposición a través de un "Popurrí de programas de TV”: http://youtu.be/hNOJwv-PtJU

\section{Resultados}

Los resultados obtenidos están principalmente basados en la percepción de los estudiantes, a través de sus respuestas a un cuestionario circularizado al finalizar el proyecto. También se toman en consideración las evidencias de aprendizaje recabadas (revistas y exposiciones) y los instrumentos de evaluación utilizados (rúbricas y coevaluaciones). Entre los resultados más significativos, cabe mencionar los siguientes:

\section{-Los estudiantes fortalecen habilidades relevantes para el adecuado ejercicio profesional de la Auditoría de cuentas.}

En concreto, estos ponen de manifiesto que el desarrollo de este proyecto les ha servido, fundamentalmente, para mejorar su capacidad de trabajar en equipo, organizarse para cumplir con los plazos fijados, gestionar el tiempo y los recursos disponibles, utilizar las TIC y comunicarse oralmente y por escrito. Todas ellas son destacadas en la normativa de referencia y la literatura previa.

\section{-Los estudiantes fomentan su actitud hacia la innovación y la creatividad.}

A este respecto, valoran sobre todo la libertad concedida a la hora de elegir la forma de exponer la revista, resaltando especialmente que se les anime a incorporar en sus presentaciones el apoyo de medios materiales y audiovisuales. Adicionalmente, los estudiantes subrayan como punto positivo que se les haya obligado a ponerse en contacto con profesionales del sector, ya que ello les acerca a la realidad empresarial y aporta un valor añadido que complementa el aprendizaje recibido en el aula a través de las clases impartidas por los profesores. 


\section{-Los estudiantes ponen en valor el esfuerzo que han realizado.}

El 51,4\% de los estudiantes toman en consideración las observaciones y comentarios realizados por los profesores en la primera entrega de la revista, procediendo a introducir cambios y llevando a cabo una segunda entrega de la revista mejorada. Esto hace que de una calificación media de 7,1 en la primera entrega (con un mínimo de 3,9 y un máximo de 9,5) se pase a una calificación media de 8,0 (con un mínimo de 5,8 y un máximo de 10). Así pues, una vez realizado el esfuerzo durante el cuatrimestre, la mayoría de los estudiantes están dispuestos a hacer un sacrificio adicional, incluso en época de exámenes, para intentar sacar un mayor rendimiento al trabajo realizado. Adicionalmente, cabe señalar que, por medio de la coevaluación, el 54,8\% de los estudiantes penaliza a alguno de sus compañeros de equipo, siendo la penalización media y máxima el 4,2\% y el 23,7\% de los puntos asignados a todo el equipo, respectivamente.

-Los estudiantes aprenden a enfrentarse a la realización de tareas diversas y de largo recorrido con apoyo del trabajo colaborativo.

La forma más común de trabajar en equipo es la de repartirse las diferentes tareas y luego ponerlo en común con los demás $(57,1 \%)$. Esto permite a los estudiantes organizarse y trabajar de tal manera que puedan cumplir los plazos previstos. Aunque la mayor parte de los estudiantes considera que la carga de trabajo que conlleva la elaboración de la revista es muy elevada (66,2\%), también se estima de forma mayoritaria que los puntos asignados a los contenidos (3 puntos sobre 10) y a la exposición (1 puntos sobre 10) son adecuados $(67,8 \%$ y $57,9 \%$, respectivamente). Además, el $84,4 \%$ afirma que si tuviera que repetir el trabajo lo haría en equipo y con los mismos compañeros y el 82,6\% considera que tener que dar a este proyecto un formato de revista le aporta un valor añadido.

\section{Conclusiones}

El proyecto desarrollado y explicado brevemente en esta comunicación se adscribe a una asignatura específica: “Auditoría de cuentas”, dentro del campo de las Ciencias Económicas y Empresariales. No obstante, su puesta en práctica es perfectamente transferible a otras asignaturas, a otras áreas de conocimiento e, incluso, a otras etapas educativas, siempre que se incorporen las adaptaciones que sean necesarias. Además, puede llevarse a cabo en colaboración con otras universidades, nacionales e internacionales, como así se ha hecho en el presente curso con un grupo de estudiantes de Contaduría Pública y Finanzas del Instituto Tecnológico y de Estudios Superiores de Monterrey y la Universidad de Colima (México).

Si bien los resultados comentados en este trabajo responden a la percepción de los alumnos sobre el proyecto planteado en el presente curso académico, cabe destacar que el mismo se viene realizando desde hace 5 años. Esto ha permitido, en base a la experiencia adquirida,

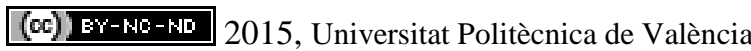


El Aprendizaje Orientado a Proyectos a través de la Elaboración de una Revista Profesional: Aplicación a la Asignatura de "Auditoría de Cuentas"

introducir progresivamente un conjunto de ajustes, como por ejemplo la incorporación de las rúbricas de evaluación, la elaboración de una guía instruccional para facilitar su desarrollo o la revisión de la ponderación del trabajo sobre la calificación global de la asignatura, que se han traducido en la obtención de un mayor valor añadido, tanto para los estudiantes como para los profesores.

Las principales limitaciones que se han tenido están relacionadas con el elevado número de estudiantes matriculados en la asignatura, que dificulta el seguimiento más individualizado de cada proyecto por parte de los tres profesores que imparten docencia. Adicional a esto, cabe señalar la disposición de las aulas universitarias, acondicionadas para dar clases magistrales.

Entre las dificultades encontradas es importante señalar la sobrecarga de trabajo que tienen los estudiantes en distintos momentos del cuatrimestre. A ello muchas veces contribuye la falta de coordinación entre profesores a la hora de encargar trabajos y realizar exámenes como parte de la evaluación continua. Por otro lado, cabe mencionar el poco peso que se le concede a la docencia entre los méritos para la promoción dentro del sistema universitario actual. Esto seguramente desincentiva el interés de muchos profesores por aplicar metodologías docentes innovadoras, que suponen una enorme carga de horas de formación, trabajo y evaluación.

\section{Referencias}

APOSTOLOU, B., HASSELL, J. M., REBELE, J. E. y WATSON, S. F. (2010). “Accounting education literature review (2006-2009)” en Journal of Accounting Education, Vol. 28, No. 3-4, pp. 145-197.

APOStOlOU, B., DORMiney, J. W., HASSELl, J. M. y WATSON, S. F. (2013). “Accounting education literature review (2010-2012)" en Journal of Accounting Education, Vol. 31, No. 2, pp. 107-161.

DAFF, L., DE LANGE, P. y JACKLING, B. (2012). “A comparison of generic skills and emotional intelligence in accounting education” en Issues in Accounting Education, Vol. 27, No. 3, pp. 627-645.

FRECKA, T. J. y RECKERS, P. M. J. (2010). "Rekindling the debate: What's right and what's wrong with masters of accountancy programs: The staff auditor's perspective” en Issues in Accounting Education, Vol. 25, No. 2, pp. 215-226.

HANCOCK, P., HOWIESON, B., KAVANAGH, M., KENT, J., TEMPONE, I. y SEGAL, N. (2010). “Accounting for the future” (pp. 54-62) en Evans, E., Burritt, R. y Guthrie, J. 
Accounting Education at a Crossroad in 2010. Australia: The Institute of Chartered Accountants in Australia.

INTERNATIONAL FEDERATION OF ACCOUNTANTS (IFAC) (2014). Handbook of International Education Pronouncements. International Accounting Education Standards Board (IAESB), June, New York.

KAVANAGH, M. H. y DRENNAN, L. (2008). "What skills and attributes does an accounting graduate need? Evidence from student perceptions and employer expectations" en Accounting and Finance, Vol. 48, No. 2, pp. 279-300.

LIN, P., KRISHNAN, S. y GRACE, D. (2013). “The effect of experience on perceived communication skills: Comparisons between accounting professionals and students" en Advances in Accounting Education: Teaching and Curriculum Innovations, Vol. 14, pp. 131152.

PAISEY, C. y PAISEY, N. J. (2010). "Developing skills via work placements in accounting: Student and employer views” en Accounting Forum, Vol. 34, No. 2, pp. 89-108.

PAN, P. y PERERA, H. (2012). "Market relevance of university accounting programs: Evidence from Australia” en Accounting Forum, Vol. 36, No. 2, pp. 91-108.

SENIK, R., BROAD, M., MAT, N. y KADIR, S. A. (2013). “Information technology (IT) knowledge and skills of accounting graduates: Does an expectation gap exist?” en Journal Pengurusan, Vol. 38, pp. 87-100.

STONER, G. y MILNER, M. (2010). "Embedding generic employability skills in an accounting degree: Development and impediments” en Accounting Education, Vol. 19, No. 1-2, pp. 123-138.

TEMPONE, I., KAVANAGH, M., SEGAL, N., HANCOCK, P., HOWIESON, B. y KENT, J. (2012). "Desirable generic attributes for accounting graduates into the twenty-first century: The views of employers” en Accounting Research Journal, Vol. 25, No. 1, pp. 41-55.

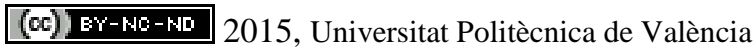

Congreso In-Red (2015) 